litativo è privo di significato clinico, anche perché per lo più, i ceppi residenti nel normale non producono enterotossine. Riportiamo dati preliminari di confronto fra isolamento quantitativo e ricerca dell'enterotossina fecale specifica.

Metodi. La conta sporale quantitativa è stata eseguita su 85 campioni (da 77 pazienti) con la metodica standard dopo shock etanolico. Per la ricerca dell'enterotossina fecale, aliquote sono state sospese in salina, centrifugate ad alta velocità, ed il sopranatante usato per il saggio di citotossicità, eseguito su tutti i campioni, e per il saggio di agglutinazione passiva inversa al lattice (PET RPLA, OXOID), eseguito su 65 campioni.

Risultati. I campioni provenivano da episodi di tossinfezioni alimentari e da diarree nosocomiali. L'uno o l'altro metodo ha rilevato l'enterotossina in 21 su 21 dei campioni con conte maggiori di $10^{6} \mathrm{CFU} / \mathrm{gr}$ feci, in 13 su 28 dei campioni negativi o con conte minori o uguali a $10^{4} \mathrm{CFU} / \mathrm{gr}$ feci ed in 26 su 36 dei campioni con conte intermedie (Chi quadro 19,116; g.d.1. $2 ; \mathrm{p}<0,0005)$. Citotossicità era dimostrabile in 34 dei 49 campioni RPLA positivi, ed in nessuno dei $16 \mathrm{cam}-$ pioni negativi o dubbi al saggio antigene (Chi quadro 20,580; g.d.1. $1 ; \mathrm{p}<0,0005)$.

Conclusioni. Abbiamo rilevato una generale concordanza tra i metodi impiegati. La connessione fra alte conte sporali e il reperto di enterotossina fecale attribuisce ad ambedue $i$ metodi una valenza diagnostica; peraltro, essendo la tossina uno dei fattori di patogenicità, riteniamo che il suo reperto sia più immediatamente significativo in senso diagnostico.

018

\section{FREQUENZA DI ISOLAMENTO DI A. CALCOACETICUS - BAUMANNII COMPLEX IN UN REPARTO DI RIANIMAZIONE}

Del Gaudio T'., Fuzio P'., Porzio M'., Lacerenza S. ${ }^{2}$, Pirronti A. ${ }^{2}$, Del Prete R. ${ }^{3}$, Miragliotta G. ${ }^{3}$

' Laboratorio Analisi P.O. Andria, AUSL BAT/I

${ }^{2}$ U.O. Rianimazione P.O. Andria, AUSL BAT/I

${ }^{3}$ Cattedra di Microbiologia, Dip. MIDIM, Univ.di Bari

Introduzione. Le infezioni ospedaliere sostenute da Acinetobacter spp. rappresentano un problema, sia per l'aumentata resistenza del microrganismo agli antimicrobici, sia perché esso è spesso causa di epidemie. Abbiamo valutato la frequenza di isolamento di Acinetobacter spp. e la sua resistenza agli antimicrobici, nel periodo Giugno 2001-Maggio 2006, in un reparto di Rianimazione dotato di 9 posti letto.

Metodi. Identificazione batterica e antibiogramma sono stati eseguiti mediante il sistema VITEK 32 (bioMérieux), il test di sensibilità alla colistina è stato effettuato mediante E-test (Biolife), mentre per la tipizzazione genica dei ceppi è stata utilizzata la metodica Pulsed Field Gel Electrophoresis (BioRAD).

Risultati. Acinetobacter calcoaceticus-baumannii complex è stato isolato da 114 pazienti ed esso era presente in 70/114 $(61.4 \%)$ nel broncoaspirato, in 35/114 (30.7\%) nel sangue. E stato inoltre isolato in $9 / 114$ diversi liquidi biologici (7.9\%). In 25/114 casi l'isolamento è stato effettuato sia da sangue che da broncoaspirato di uno stesso paziente.

I ceppi isolati hanno mostrato debole sensibilità ai carbapenemici (Imipenem 24.5\%; Meropenem 39.4\%) alla Ticarcillina+Acido clavulanico (22.8\%), mentre la resisten- za a $\beta$-lattamici, cefalosporine, aminoglucosidi, chinoloni, nitrofurantoina, tetraciclina, trimetoprim-sulfametossazolo risultava superiore all' $80 \%$. Per contro la colistina mostrava una sensibilità del 100\%.

Conclusioni. Nonostante le misure di barriera adottate, A. calcoaceticus-baumannii complex è stato isolato con elevata frequenza nel periodo considerato ed almeno in tre occasioni ha infettato un numero di pazienti superiore a quello solitamente osservato. In particolare, nel marzo 2006, tale microrganismo è stato isolato da 5 pazienti; la tipizzazione genica eseguita su 4 campioni ha dimostrato che gli isolati appartenevano ad uno stesso ceppo, che esibiva multiresistenza a tutte le classi di antibiotico succitate, mentre si rivelava sensibile alla colistina. I prelievi ambientali, eseguiti per individuare la fonte di infezione, davano esito negativo. La situazione si è andata normalizzando, elevando il grado di attenzione nell'applicazione delle barriere tra paziente e paziente e trattando quelli infetti con colistina. Dai dati ottenuti si può ipotizzare che la principale via di accesso di A. calcoaceticus-baumannii complex sia quella aerea. Inoltre la nostra esperienza dimostra l'importanza che in queste infezioni riveste la trasmissione per contatto, soprattutto in quei reparti con pazienti infetti lungodegenti e con elevata pressione antibiotica selettiva.

\section{9}

\section{UN CASO DI SEPSI DA S. PNEUMONIAE POST-SPLENECTOMIA: RIFLESSIONI}

\author{
Dono M. ' ,Artioli S. ${ }^{2}$, Amodeo C. ${ }^{3}$, Derchi A. ${ }^{3}$, Santini M. ${ }^{3}$, \\ Via F. ', Roncella S. ${ }^{4}$, Battolla E'. \\ 'U.O. Analisi; \\ ¿U.O. Malattie Infettive; \\ 3U.O. Anestesia e Rianimazione; \\ ${ }^{4}$ U.O. Anatomia ed Istologia Patologica, Osp. S. Andrea, La Spezia.
}

Introduzione. Gli individui splenectomizzati risultano piu' vulnerabili alle sepsi causate da batteri capsulati. I sintomi iniziali spesso sono non-specifici e possono, in realtà, coincidere con danni fisiologici irreversibili. Infatti, il tasso di mortalità è molto elevato.

Noi riportiamo un caso di sepsi da S. pneumoniae in un paziente che ha subito una splenectomia circa 10 anni fa. La presentazione di questo caso offre sia la possibilità di discutere il percorso diagnostico in emergenza sia lo spunto per una riflessione sulla prevenzione delle sepsi nei pazienti splenectomizzati.

Metodi. Il tampone faringeo di un paziente arrivato in pronto soccorso (PS) con rush cutaneo, viene inviato in urgenza in laboratorio per la colorazione GRAM. Viene rilevata la presenza di rarissimi diplococchi GRAM positivi.

Successivamente, su segnalazione di questo referto, viene inviato al laboratorio un campione di urina per l'analisi dell'antigene pneumococcico che risulta fortemente positivo.

Risultati. Un paziente di 43 anni arriva in PS con una grave compromissione multiorgano. I tests di laboratorio sono compatibili con una sepsi da S. pneumoniae in paziente splenectomizzato. I livelli elevati di procalcitonina $(>10 \mathrm{ng} / \mathrm{ml})$ confermano la sepsi severa. La TAC toracica rivela piccoli focolai multipli in entrambi i polmoni, di probabile origine pneumococcica.

Conclusioni. La prevenzione per il management degli indi- 
vidui senza milza comprende: la vaccinazione, la profilassi antibiotica e l'informazione dei pazienti.

Tuttavia, nonostante sia molto importante ridurre il rischio di sepsi in tali pazienti, in pratica queste misure preventive non vengono quasi mai proposte. Infatti, il nostro paziente, non consapevole di essere un individuo ad alto rischio di sepsi, aveva ignorato i pochi segnali che la polmonite pneumococcica aveva dato. E' molto importante allora, coinvolgere i pazienti sul rischio settico dopo la splenectomia soprattutto con l'informazione (e l'aiuto del clinico); inoltre, nel caso di una sospetta sepsi, è fondamentale l'anamnesi del paziente per improntare un percorso diagnostico mirato, soprattutto in urgenza.

020

\section{VALUTAZIONE DEL NUOVO SISTEMA AUTOMATICO “VIDIA" PER LA RICERCA DI ANTICORPI ANTI TOXOPLASMA E ROSOLIA}

\author{
Ferraironi M., Martinelli D. e Cipriani P.
}

Il Facoltà di Medicina e Chirurgia Università La Sapienza Roma - Servizio di Microbiologia, Azienda Ospedaliera S. Andrea, via di Grottarossa 1035, 00189 Roma

Introduzione. Il sistema VIDIA (bioMérieux) è un nuovo strumento automatico con gestione della provetta primaria, concepito per semplificare la routine e per implementare il livello di rintracciabilità dei dati.

Nel presente lavoro abbiamo valutato le performance dei test VIDIA per gli anticorpi anti-Toxoplasma ed anti-Rosolia confrontandola con il sistema attualmente in uso nel nostro laboratorio AxSym - Abbott.

Metodi. Sono stati analizzati complessivamente 200 campioni appartenenti ad una popolazione mista, per determinare TOXO IgG e IgM, e RUB IgG e IgM. I campioni con risultati discordanti sono stati ripetuti con il metodo VIDA e, inoltre, sono previsti ulteriori approfondimenti utilizzando il test di riferimento Toxo Isaga.

È stata inoltre valutata la ripetibilità intra-serie e la riproducibilità inter-serie del test VIDIA TOXO IgG e VIDIA RUB IgG secondo i suggerimenti dell'NCCLS, utilizzando 2 pool di sieri a 4 diverse concentrazioni.

Risultati. Globalmente si è riscontrata una prevalenza pari a circa il $19 \%$ per Toxo IgG, il 3\% per Toxo IgM, il $77 \%$ per Rosolia IgG e 1' 1\% per Rosolia IgM.

La concordanza tra i metodi a confronto è stata del 100\% per il Toxo IgG, 98\% per Toxo IgM, 99\% per Rosolia IgG e 99\% per Rosolia IgM.

Sui risultati discordanti sono in corso approfondimenti diagnostici per stabilire se tale discordanza sia da imputare solo al metodo usato o possa dipendere anche dallo stato immunologico dei pazienti soprattutto se si tratta di donne in gravidanza.

La precisione intra-serie e la ripetibilità inter-serie $(\mathrm{CV} \%)$ dei test VIDIA TOXO IgG e VIDIA RUB IgG erano comprese tra 2 e $4 \%$ circa.

Conclusioni. Dai risultati emerge un elevato livello di concordanza tra il sistema VIDIA e il metodo AxSym attualmente in uso. Si rileva inoltre un elevatissimo grado di precisione intra-serie ed inter-serie dei test VIDIA.

\section{1}

\section{LEPTOSPIROSI CON ISOLAMENTO COLTURALE POSITIVO}

\author{
Franzin L.', Cabodi D.', Ciarrocchi S. ${ }^{2}$,
}

Pinto A. ${ }^{2}$, Ciceroni L. ${ }^{2}$

' Laboratorio "Ricerca Speciale Microbiologica",

Dipartimento Diagnostica di Laboratorio,

Ospedale Amedeo di Savoia,

Corso Svizzera 164, 10149 Torino.

${ }^{2}$ Centro Nazionale per le Leptospirosi,

Dipartimento di Malattie Infettive, Parassitarie

ed Immunomediate, Istituto Superiore di Sanità,

Viale Regina Elena 299, 0016 I Roma.

Introduzione. Leptospirosi è una malattia di difficile diagnosi di laboratorio. L'esame colturale fornisce una prova diretta e definitiva dell'infezione, non sempre ottenibile con esami sierologici. Nella leptospirosi a rapido decorso ed esito infausto la coltura è l'unico metodo in grado di consentirne la diagnosi. Inoltre la tipizzazione dei ceppi isolati è di rilevante importanza epidemiologi$\mathrm{ca}$, in quanto permette di identificare il serovar infettante, di conoscerne la distribuzione geografica e talvolta di stabilire la sorgente di infezione o il reservoir animale. Riportiamo un caso di leptospirosi fulminante, diagnosticato con coltura, per il quale è stato definito il serovar infettante mediante tecniche di tipizzazione sierologica e molecolare.

Metodi. Paziente: soggetto maschio di 35 anni con febbre, ittero, insufficienza renale acuta, shock cardiocircolatorio. Circostanza presunta del contagio: caduta accidentale con automobile in un canale. Comparsa dei sintomi: una settimana dopo l'incidente. Penicillina G è stata somministrata dal $4^{\circ}$ giorno dall'inizio dei sintomi. Il paziente è deceduto il giorno successivo. Metodi: La ricerca di anticorpi è stata eseguita su siero prelevato al $4^{\circ}$ giorno di malattia mediante la reazione di agglutinazione microscopica (MAT) eseguita con 20 ceppi rappresentativi di 19 serovar e 14 sierogruppi. L'esame colturale è stato eseguito da sangue eparinato, da urina e da campioni autoptici (rene, polmone, milza, miocardio, fegato). Risultati. La sierodiagnosi ha evidenziato anticorpi antiLeptospira 1:320 verso il serovar Poi. La coltura è risultata positiva da sangue e da urina. Entrambe gli isolati sono stati identificati come serovar Icterohaemorrhagiae (sierogruppo Icterohaemorrhagiae) mediante MAT eseguita con antisieri di coniglio ed anticorpi monoclonali. I risultati della tipizzazione sierologica sono stati confermati mediante analisi del DNA genomico eseguita con PFGE.

Conclusioni. I risultati ottenuti confermano l'importanza dell'esame colturale nella diagnosi di leptospirosi e che solo attraverso la tipizzazione degli isolati è possibile identificare con certezza il serovar di appartenenza della leptospira infettante. 